\title{
Towards Understanding the Role of Carbon Atoms on Transition Metal Surfaces: Implications for Catalysis
}

\author{
Biel Martínez, Oriol Piqué, Hèctor Prats, Francesc Viñes*, Francesc Illas \\ Departament de Ciència de Materials i Química Física \& Institut de Química Teòrica i \\ Computacional de la Universitat de Barcelona (IQTCUB), c/ Martí i Franquès, 1-11, \\ Barcelona, Spain.
}

*Corresponding author: francesc.vines@ub.edu

\begin{abstract}
Carbon moieties, in a low coverage regime being reduced to $\mathrm{C}$ adatoms, are a rock-inthe-shoe for heterogeneously catalyzed processes involving carbon-containing species. Their presence affects the performance of Transition Metal (TM) based industrial catalysts, often resulting in poisoning. Recent studies on the $\mathrm{C}$ adatom thermodynamic stability revealed that both surface and subsurface $\mathrm{C}$ atoms may coexist, indicating additional poisoning mechanisms, yet also new catalytic promoting mechanisms. The present work provides a systematic study of the potential dynamic relevance of such subsurface $\mathrm{C}$ atoms in the most stable (111) surface of all fcc TMs at low C coverages. This relies on evaluating the composition at thermodynamic equilibrium and the time scale of the different involved processes by means of Density Functional Theory (DFT) and kinetic Monte Carlo $(k M C)$ simulations, respectively. These DFT and kMC simulations highlight the relevant role of subsurface $\mathrm{C}$ atoms for $\mathrm{Ag}$ and $\mathrm{Pd}$, and a fast $\mathrm{C}$ mobility for $\mathrm{Au}$ and $\mathrm{Pt}$, which might be important factors contributing to poisoning or opening new reactive path mechanisms, especially relevant at high temperature working conditions.
\end{abstract}


Graphical Abstract

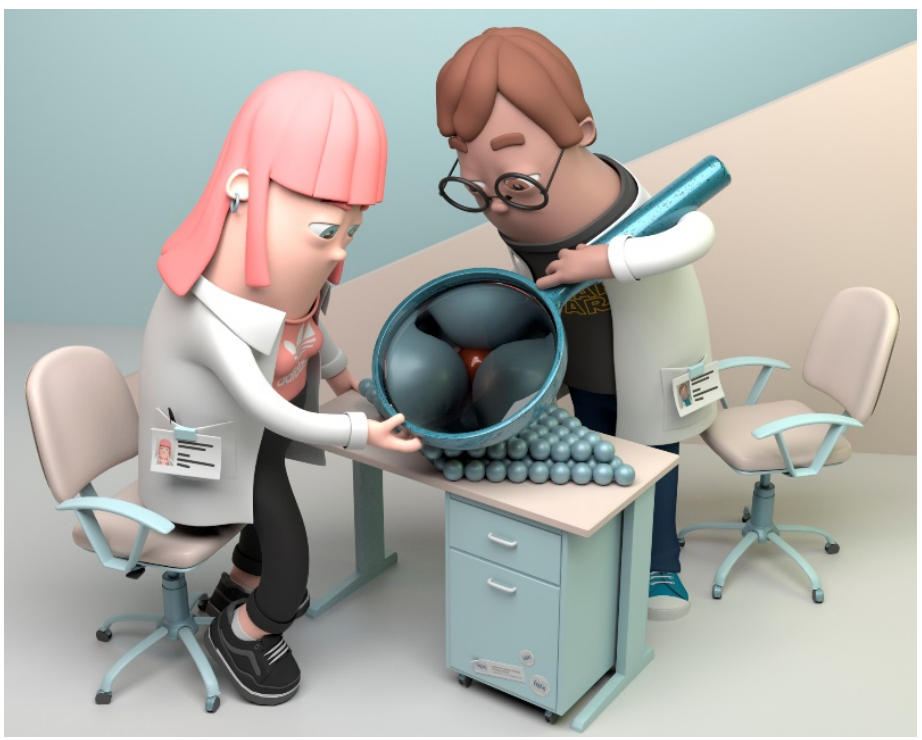




\section{Introduction}

Transition Metals (TMS) are crucial in many heterogeneously catalyzed industrial chemical processes with huge relevance in the modern mankind society development. The production of ammonia, for instance, is to date mostly carried out through the Haber-Bosch process, using Fe as a catalyst [1]. Specifically, due to their established moderate chemical activity [2], TMs deliver a good compromise between affordable reaction energy barriers and moderate binding strengths of the involved reaction species -i.e., fulfilling Le Sabatier principle - and, hence, are broadly employed in heterogeneous catalysis [3]. Nevertheless, research endeavors are continuously undertaken to develop novel or improved catalysts involving TMs as indispensable components, either as active phase, as supports, forming alloys, or even in the limit of isolated single atoms, the so-called Single Atom Catalysts (SACs). Among the TMs used in heterogeneous catalysis, those with face-centered cubic $(f c c)$ crystal structure are prominent, e.g. in the hydrogen production, catalyzed by $\mathrm{Ni}$ [4], the generation of nitric acid through the Ostwald process using $\mathrm{Pt}$ or $\mathrm{Rh}$ as catalysts [5], methanol synthesis using large supported $\mathrm{Cu}$ nanoparticles [6], or the partial oxidation of methane to carbon monoxide, achievable using $\mathrm{Ni}, \mathrm{Ru}, \mathrm{Rh}, \mathrm{Pd}$, Ir, or Pt [7].

Many of the aforementioned industrially relevant processes involve carboncontaining species. In fact, all the organic and fine chemistry industries handle, generically, carbon rich molecules. During the formation or breaking of chemical bonds, some intermediate species can eventually escape from the sought catalytic cycle through side secondary reactions, resulting in $\mathrm{C}_{\mathrm{n}}$ moieties attached to the catalyst surface. These carbon residues can eventually lead to coke formation, which would poison the catalyst by blocking the active surface metal sites [8]. This is indeed a frequent problem on $f c c$ TM surfaces, where poisoning by carbon species have been reported for a wide variety of catalytic processes [9-11], thus reducing or even jeopardizing the catalytic activity over time, and ultimately, requiring costly catalyst regeneration steps.

Typically, poisoning occurs at high carbon coverage, $\theta_{\mathrm{C}}$, where different complex structures are found going from carbon filaments to graphene, graphite, or amorphous carbon $[12,13]$. At very low $\theta_{\mathrm{C}}, \mathrm{C}$ remains mostly isolated, normally as surface adatoms [13]. Recent works studied the thermodynamic and kinetic stability of such $\mathrm{C}$ atoms on most-stable (111) surfaces of $f c c$ TMs and realistic nanoparticle models, revealing for a number of the studied cases a rather unexpected higher stability at subsurface sites 
$[14,15]$. So far, this extended surprising feature has not been taken into account in the computational models previously used to simulate catalytic process on such surfaces. The consideration of the presence of subsurface carbon might well lead to a change of paradigm in our understanding of the catalytic activity and reactivity of a variety of metal surfaces that can experience this phenomenon. Indeed, there are experimental and theoretical works that already underline the pivotal role of such $\mathrm{C}$ species on tuning the catalytic activity, reactivity, and selectivity of Pd catalysts [16,17].

Here we go one step further investigating the dynamic aspects of surface/subsurface C stability on all $f c c$ TM (111) surfaces. To this end we perform kinetic Monte Carlo $(k M C)$ simulations, with rates obtained from Density Functional Theory (DFT) based calculations, aimed at gaining information of the surface structure under realistic working conditions over time. This information is likely to be relevant for the determination of the potential role of surface or subsurface $\mathrm{C}$ species, aside, with the aim to ascertain whether the lifetime of such species falls in the reaction rate time-lapse, and, therefore, should be considered as a potential catalytic role-player.

\section{Computational Details}

The DFT based calculations have been carried out for suitable periodic models of the $f c c$ TM (111) surfaces (TM = Ni, Cu, Rh, Pd, Ag, Ir, Pt, and Au) using the Vienna $A b$ Initio Simulation Package (VASP) [18], and employing the Perdew-Burke-Ernzerhof $(P B E)$ form of the exchange and correlation functional [19], shown to be one of the most accurate choices for a wide variety of TM systems and properties, including the atomic and electronic structure of bulk and low Miller index surfaces [20-23]. The (111)

surface has been chosen since it is the most stable surface and, according to the Wulff construction minimizing the surface energy [21,24], the most exposed in large supported nanoparticles in the real catalysts.

The adsorption of atomic $\mathrm{C}$ has been studied on the highest symmetry sites, including top, bridge, and three fold hollow sites $-f c c$, having a subsurface metal atom two layers underneath, and hexagonal close-packed ( $h c p$ ), having a metal atom in the immediate subsurface layer-, being hollow sites the most preferred by $\mathrm{C}$ species. As far as subsurface sites are concerned, several non-equivalent sites have been sampled with the $\mathrm{C}$ atom in between the first and second atomic metal layers, finding that the only relevant adsorption sites are the $f c c$ subsurface -also known as octahedral 
subsurface site, oss - and the hcp subsurface - known as tetrahedral subsurface site, $t s s-$. The energy barriers for diffusion, $E_{\mathrm{b}}$, of $\mathrm{C}$ species along the (111) surface, along the (111) subsurface, and in between the surface and subsurface regions have been obtained using the Climbing Image Nudged Elastic Band (CI-NEB) method [25].

To represent the TM (111) surfaces, a $p(3 \times 3)$ supercell with six atomic layers, containing a total of 54 metal atoms, has been used. The three uppermost layers have been allowed to fully relax, whereas the three bottommost layers were fixed at optimized bulk crystal positions to simulate bulk rigidity. Such models are wide enough so to deliver converged results up to few tenths of an $\mathrm{eV}$ - below c.a. $0.04 \mathrm{eV}$ - for absorption and adsorption energies [15]. The valence electron density was expanded in a plane wave basis set, with a kinetic energy cutoff of $415 \mathrm{eV}$. In the self-consistent field method, a suitable smearing algorithm has been used to enhance the convergence of the self consistent field procedure, yet final energies have been extrapolated to $0 \mathrm{~K}$. The reported energies account for the Zero Point Energy $(Z P E)$ term, calculated within the harmonic approximation. The frequency calculations have been computed from diagonalization of the corresponding block of the Hessian matrix with elements computed from finite displacements of analytic gradients; a value of $0.01 \AA$ was considered for all the atoms relaxed during the structural optimization or the Transition State (TS) search location. The sampling of the reciprocal space has been carried out following a $3 \times 3 \times 1$ Monkhorst-Pack k-points grid [26]. The $E_{\mathrm{b}}$ for the sinking-emerging processes have been extracted from literature [14], yet here including the ZPE term.

A Python-based kMC code has been written to propagate the system correctly from configuration to configuration by means of the widely used rejection-free Variable Step Size Method (VSSM) [27]. The different TM (111) surfaces are represented in the kMC framework as a two-dimensional periodic lattice, where each adsorption site corresponds to a point in the lattice. The latter is built by replicating $\mathrm{L} \times \mathrm{L}$ times the $p(1 \times 1)$ primitive unit cell, which contains two surface sites (i.e., one $f c c$ and one $h c p$ ), and two subsurface sites (i.e., one oss and one tss), as shown in Figure 1. Periodic boundary conditions were included in the $\mathrm{kMC}$ simulations to emulate the extended character of the surface at the atomic scale. As the purpose here is to study the low $\theta_{\mathrm{C}}$ real situations, the $\mathrm{kMC}$ simulations have been performed in the $\theta_{\mathrm{C}}$ range of $1-9$ at $\%$ and a temperature, $T$, range of $300-700 \mathrm{~K}$. Note that $\theta_{\mathrm{C}}$ at $\%$ is defined as the percentage of carbon atoms per lattice surface lattice sites, being these the sum of the 
$h c p$ and $f c c$ hollows in the unit cell. At this low-coverage regime, the effect of possible lateral interactions, not accounted in this $\mathrm{kMC}$ model, is irrelevant, even more when they have been seen to be rather anecdotic, if any, during the simulations, as just very rarely $\mathrm{C}$ atoms are located vicinal to each other. A total of eight types of diffusion processes have been considered for the present $\mathrm{kMC}$ simulations, which involve the reversible surface and subsurface diffusions from $f c c$ to $h c p$ and from tss to oss, respectively, as well as the reversible sinking/emerging processes from hcp to tss and from $f c c$ to $o s s$.

For diffusion processes at solid surfaces, the adsorbed species only contribute to the vibrational partition function, and the rate constant can be obtained from Transition State Theory (TST) as:

$$
k=\frac{k_{B} T}{h} \frac{Q_{T S}}{Q} e^{-\frac{E_{b}}{k_{B} T}}
$$

where $E_{\mathrm{b}}$ is the energy barrier including the ZPE term, $h$ the Planck constant, $k_{\mathrm{B}}$ the Boltzmann constant, and $Q_{\mathrm{TS}}$ and $Q$ the vibrational partition functions at the TS and at the initial state, respectively, which can be computed as:

$$
Q=\prod_{i=1}^{3 N} \frac{1}{1-e^{-\frac{v_{i} h}{k_{B} T}}}
$$

for energies already including the ZPE term, where $N$ is the number of relaxed atoms, and the product runs for the $3 \cdot N$ vibrational frequencies, $v_{i}$. Note that our reaction model involves diffusion processes with very dissimilar $E_{\mathrm{b}}$ values. In order to speed up the $\mathrm{kMC}$ simulations, the difference in time scales has been handled by manual scaling the rate constants of the fastest processes by some appropriate factor $\alpha<1$, while ensuring that this scaling does not alter the final outcome of the simulation. This solution has been successfully applied in many previous kMC studies [28-31]. Here, we applied $\alpha$, so that $\log _{10}(\alpha)=-6,-2$, and -3 for surface diffusion in $\mathrm{Cu}, \mathrm{Ni}$, and $\mathrm{Rh}$, respectively; and $\log _{10}(\alpha)=-2$ for Pt $h c p \leftrightarrow t s s$ processes. Additional details regarding the manually scaling and the validation of the $\mathrm{kMC}$ code are provided in the Supplementary Material (SM).

The chosen $\theta_{\mathrm{C}}$ is simulated by initially repeating the lattice unit cell and adding an appropriate number of $\mathrm{C}$ atoms at random positions, see Table 1. All kMC simulations have been carried out until the system reached the equilibrium, plus $5 \cdot 10^{6}$ extra steps for production, from which the overall macroscopic properties have been extracted. First, 
the mean percentage of $\mathrm{C}$ atoms at each site have been determined. Moreover, the average waiting time between an emerging event and its subsequent emerging in a given surface area through either $f c c$ or $h c p$ site, $\bar{t}_{e m}$, in units of time per surface area, has been computed as the quotient between the total production time, $t_{p}$, and the number of emerging processes, $n_{e m}$, occurred through a $f c c$ or an $h c p$ site, times the area $A$ of the simulation cell:

$$
\bar{t}_{e m}=\frac{t_{p}}{\left(n_{e m}\right) A}
$$

Another interesting property is the average lifetime of a $\mathrm{C}$ atom in a given region, $l t_{r}$, where $r$ relates to being surface or subsurface, or on a given site type, $l t_{j}$, where $j=h p c$, $f c c$, tss, or oss, which can be calculated from the kMC simulations as:

$$
\begin{aligned}
& l t_{r}=\frac{1}{n_{C}} \sum_{i=1}^{n_{C}} \frac{t_{r}^{i}}{n_{r}^{i}} \\
& l t_{j}=\frac{1}{n_{C}} \sum_{i=1}^{n_{C}} \frac{t_{j}^{i}}{n_{j}^{i}}
\end{aligned}
$$

where $n_{C}$ is the number of carbon atoms, $t_{r}^{i}$ and $t_{j}^{i}$ are the time spent for the $i^{\text {th }}$ atom in the region $r$ and the site type $j$, respectively, and $n_{r}^{i}$ and $n_{j}^{i}$ are the number of processes experienced by the $i^{\text {th }}$ atom that drove it to the region $r$ and the site type $j$, respectively. Obtaining these aforementioned properties allows one to reach not only a complete analysis of the equilibrium state but also a deeper analysis on the time scales of the equilibrium fluctuations, that could very well be a key factor on the surface chemistry of a significantly carbided surface.

\section{Results and Discussion}

The relative energetic stability of $\mathrm{C}$ at the different adsorption sites, as well as the energy barriers for sinking/emerging processes, have been taken from the previous work by Piqué et al. [14]. Here we have performed additional DFT calculations at the same level of theory to obtain the energy barriers for surface and subsurface diffusion, as well as to include the ZPE term, which was previously neglected. Figures 2 and 3 show the energy profiles for all diffusion processes along with the energy barriers for both forward and reverse directions, while values of the relative stability of $\mathrm{C}$ atoms on the different adsorption sites are reported in Table S1 of the SM. The most stable region for 
a $\mathrm{C}$ atom on $\mathrm{Ni}, \mathrm{Cu}, \mathrm{Pd}$, and $\mathrm{Ag}$ is subsurface, specifically in oss, understandable just in terms of cavity space as compared to tss. In fact, oss is found to be more stable than tss for all the studied transition metals except for Pt and Au. On the contrary, C prefers the surface region on $\mathrm{Rh}, \mathrm{Ir}, \mathrm{Pt}$, and $\mathrm{Au}$, with both $f c c$ and $h c p$ sites featuring similar adsorption energies. As one moves along a TM series and up a group in the periodic table, the metal atomic radii decreases, and the $\mathrm{C}$ subsurface stability compared to the surface stability increases, since metals with smaller radii allow for extra space within their voids.

The $E_{\mathrm{b}}$ values for surface diffusion range from 0.04 for $\mathrm{Cu}$ to $0.79 \mathrm{eV}$ for $\mathrm{Pt}$, suggesting a likely surface $\mathrm{C}$ adatoms mobility at working conditions. Surprisingly, subsurface diffusion does not imply much larger energy barriers, which range from 0.39 for $\mathrm{Au}$ to $1.18 \mathrm{eV}$ for $\mathrm{Ni}$. Note that the diffusion across the subsurface region implies an elongation of the three-fold hollow sites metal-metal bonds involved in the $\mathrm{C}$ crossing pathway. At first sight, one would argue that this deformation has an energy cost that could largely increase the $E_{\mathrm{b}}$ values. However, the TS structure appears to be stabilized by the surrounding metal atomic coordination shell, which compensates the structural distortion destabilization. Interestingly, $\mathrm{C}$ atoms diffuse more easily when the surface chemical activity is small, this is, when moving along a TM series and down a group, being weakly bonded to a specific site, which happens to be a common trend of adsorbed diffusing species on transition metals [32].

In general, sinking/emerging processes present higher $E_{\mathrm{b}}$ compared to surface/subsurface diffusions, as the former imply an increase of the surface metalmetal bonds of the three surface metal atoms conforming the hollow sites along the $\mathrm{C}$ atom crossing pathway $[15,33]$, but without being much stabilized by coordination. The sinking energy barriers range from 0.26 to $1.80 \mathrm{eV}$ for $\mathrm{Au}$ and $\mathrm{Ir} h c p \rightarrow t s s$ cases, respectively. On the other hand, the emerging energy barriers range from only 0.07 to $1.45 \mathrm{eV}$, both belonging to $\mathrm{Ni}$, but for $t s s \rightarrow h c p$ and $o s s \rightarrow f c c$, respectively. In general, the sinking/emerging energy barriers decrease while going down a group of the periodic table, due to a combination of larger interstices and malleability of the metals [15]. Note that the high subsurface $\mathrm{C}$ stability does not necessarily imply a smaller $E_{\mathrm{b}}$ for the $\mathrm{C}$ sinking. For instance, $\mathrm{C}$ is very stable at the oss site of both $\mathrm{Ni}$ and $\mathrm{Ag}$, but their corresponding sinking energy barriers are 0.84 and $0.43 \mathrm{eV}$, respectively. Thus, dynamic simulations are necessary to shed light into whether the sinking/emerging $E_{\mathrm{b}}$ 
values are determining, i.e. large enough to prevent the exchange of $\mathrm{C}$ atoms between surface and subsurface sites under working conditions.

To have a deeper insight into the overall dynamic process of $\mathrm{C}$ atoms at these surfaces, we carried out kMC simulations under operando conditions, and calculated several macroscopic properties such as the percentage of total $\mathrm{C}$ at the surface and subsurface regions, with results reported in Figure 4. The kMC simulations show that, at $300 \mathrm{~K}, \mathrm{C}$ atoms are almost only at the surface for $\mathrm{Rh}$, Ir, Pt, and $\mathrm{Au}$; and almost only in the subsurface for $\mathrm{Ni}, \mathrm{Cu}, \mathrm{Pd}$, and $\mathrm{Ag}$, in agreement with the relative energetic stability predicted by the DFT calculations. Indeed, the $100 \%$ surface situation is found for Ir, whereas a $100 \%$ of subsurface $\mathrm{C}$ is found for $\mathrm{Pd}$. Hence, in the low $\theta_{\mathrm{C}}$ regime, the large number of empty sites enables the location of all $\mathrm{C}$ atoms in their thermodynamically most stable adsorption sites. Tables S2-S9 of the SM summarize the percentage of C atoms in all four different types of adsorption sites at all temperature values considered. The kMC simulations allow us to keep track of the preferred paths for the $\mathrm{C}$ atoms within the lattice. In general, there is a drastically larger preference for the sinking/emerging processes to occur through a $h c p$ site, which is also in agreement with the smaller $E_{\mathrm{b}}$ values, see Figure 3 . The reason is that $\mathrm{C}$ at $t s s$ sites is generally less stable than at oss, and, hence, features lower emerging barriers. In addition, the subsurface metal atom stabilizes the TS for sinking through a coordination effect. Overall, the $\mathrm{C}$ motion is mainly composed by diffusion steps either at the surface or across subsurface, connected through sinking/emerging processes involving $h c p$ and tss sites. Thus, the occupation of oss sites, which are the most stable sites in the subsurface, is not reached through a direct sinking form a $f c c$ surface site, but rather from a $h c p \rightarrow t s s$ sinking followed by a $t s s \rightarrow$ oss subsurface diffusion.

The effect of $\theta_{\mathrm{C}}$ on the amount of $\mathrm{C}$ present at the surface or subsurface sites is negligible, even for the $\theta_{\mathrm{C}}$ of 9 at $\%$. However, the effect of temperature is relevant in some of the studied surfaces, leading to an increase of the $\mathrm{C}$ population in the less stable sites. This effect is especially prominent for $\mathrm{Ag}$ and Pt. In the case of $\mathrm{Ag}$, there is a decrease of subsurface $\mathrm{C}$ concentration by increasing the temperature, which represents up to a $7 \%$ of the total $\mathrm{C}$ released to the surface at $700 \mathrm{~K}$. This suggests that upon annealing, C containing Ag (111) samples would feature a segregation of C towards the surface, rather than increasing solubility of $\mathrm{C}$ within the material. In the case of Pt, the amount of $\mathrm{C}$ in subsurface sites increases from $2 \%$ at $300 \mathrm{~K}$ to $13 \%$ at $700 \mathrm{~K}$, thus 
becoming an important factor to take into consideration when dealing with Pt catalysts in presence of $\mathrm{C}$ moieties and also when modeling organic chemistry reactions on $\mathrm{Pt}$ (111) terraces at working conditions. Note that these unexpected results cannot be anticipated by inspection of the DFT energy profiles alone, and may be very relevant in the catalytic activity of these transition metal surfaces at high working temperatures.

The presence of subsurface $\mathrm{C}$ can affect the surface chemical activity in different ways, such as increasing or reducing the adsorption strength of particular molecules. The subsurface C stability in Pd (111) at all operating conditions explains the observed $\mathrm{C}_{1}$ promoting role of $\mathrm{Pd}$ catalysts in alkyne dehydrogenation reactions [34,35]. Moreover, for cases where $\mathrm{C}$ atoms are preferentially located at the surface, the sinking of $\mathrm{C}$ atoms to the subsurface might be followed by their diffusion and posterior emerging to other regions, either $\mathrm{C}$-containing or $\mathrm{C}$-free. This process can be enhanced with the temperature, especially in the case of $\mathrm{Pt}, \mathrm{Rh}$, and $\mathrm{Au}$. In particular, this can explain the double-layer model as detected on Pt (111) [36], consisting of a graphenelike layer, with $\mathrm{C}$ atoms connecting it to the $\mathrm{Pt}(111)$ surface [37]. Alternatively, for cases where $\mathrm{C}$ prefers to be in the subsurface region, the surface would be apparently clean, yet $\mathrm{C}$ atoms would remain trenched at the oss sites. Apart from the possible perturbing effect on the expected surface activity, their possible emergence could imply these $\mathrm{C}$ atoms being involved in the on-going reaction, a point which may well be the case for $\mathrm{Ag}$, and for $\mathrm{Ni}$ and $\mathrm{Cu}$ to a lesser extent, at high temperatures.

In order to quantify the occurrence probability of processes involving emerging $\mathrm{C}$ atoms, we have to consider the emergence feasibility and the lifetime of $\mathrm{C}$ species at the surface and on subsurface sites. Figure 5 displays the average lifetime of $\mathrm{C}$ atoms at surface and subsurface regions in the range of studied temperatures and $\theta_{\mathrm{C}}=1$ at $\%$. Note that, as stated above, results for this quantity are independent of the $\mathrm{C}$ coverage. Obviously, longer lifetimes are obtained for the most stable regions, which are rarely below the $\mu$ s even at $700 \mathrm{~K}$. This indicates that, once the system is at equilibrium, there are very few sinking and emerging processes. Interestingly, when they occur, the lifetime of the $\mathrm{C}$ on the new, less stable situation, is generally still in the range of the ps to ms, which cannot be neglected as surface reaction steps are comprised at these time scales. Note that both $l t_{\text {surf }}$ and $l t_{\text {sub }}$ decrease when increasing the temperature and while going down a group, in agreement with the decrease in sinking/emerging energy barriers. 
Let us analyze in more detail the systems for which $\mathrm{C}$ prefers a subsurface site; i.e. $\mathrm{Ni}, \mathrm{Cu}, \mathrm{Pd}$, and $\mathrm{Ag}$. All of them present $l t_{\text {surf }}$ below $1 \mathrm{~s}$, and even below $1 \mathrm{~ms}$ at $700 \mathrm{~K}$. Thus, when the exposure time is larger than $l t_{\text {surf }}$, one would expect that all $\mathrm{C}$ atoms go subsurface. Since catalytic cycles may operate well beyond this ms time scale, one can confirm that a migration of $\mathrm{C}$ from surface to subsurface sites in these metal surfaces will occur under realistic operando catalytic conditions, which may introduce changes in the performance of the catalyst. In addition, the effect of surface $\mathrm{C}$ does not have to be misregarded, given that reaction steps occur in the fs to ms scales frame where asgenerated surface $\mathrm{C}$ atoms can be present.

Figure 6 shows the mean lifetimes for the $\mathrm{C}$ atoms on the different types of adsorption sites, as well as the average waiting time between two consecutive emerging processes, $\bar{t}_{e m}$, at 300 and $700 \mathrm{~K}$, and $\theta_{\mathrm{C}}=1 \mathrm{at} \%$. The obtained results for all the other studied operating conditions can be found in Tables S10-S17 of the SM, together with some hints to ease the interpretation of Figure 6. In general, the simulations show that $\mathrm{C}$ lifetimes at particular sites are many orders of magnitude lower than lifetimes at surface or subsurface, indicating that intra-layer diffusion processes are dominant. This alters the static image that one could get from the systems when analyzing Figure 4 and suggests that there is a continuous diffusion of $\mathrm{C}$ atoms within the same metal layer. Before going into details for the different cases, a rough analysis of the results in Figure 6 predicts that $\mathrm{C}$ at $\operatorname{Ir}$ (111) can be regarded as an example of a rather static system, with large waiting times, and long lifetimes on specific times, almost as much as the overall surface or subsurface lifetimes. On the other hand, Pt and Au show short waiting times, and comparable high and easy mobilities. Finally, $\mathrm{Ni}, \mathrm{Cu}, \mathrm{Rh}, \mathrm{Pd}$, and $\mathrm{Ag}$ are in between the two previous scenarios. These behaviors quite correlate to what is expected from the energy profiles and energy barrier values shown in Figures 2 and 3.

For the systems where carbon is found to more stable at subsurface, namely $\mathrm{Ni}, \mathrm{Cu}$, $\mathrm{Pd}$, and $\mathrm{Ag}, \bar{t}_{e m}$ is in the ns and $\mathrm{s}$ range, normally ns for $t s s \rightarrow h c p$ transitions. This couples to $l t_{\text {surf }}$ values ranging from fs to ns, large enough to allow the interaction of the emerging $\mathrm{C}$ with available adsorbates with possible concomitant effects on the ongoing surface catalyzed process. Although the emergence of $\mathrm{C}$ at $300 \mathrm{~K}$ is in the seconds time frame, $\bar{t}_{e m}$ clearly drops to $\mu$ s or ns when the system is annealed to $700 \mathrm{~K}$, even though this reduces $l t_{\text {surf }}$, which still is slightly below the ms time scale. This result indicates that, on the average, one can expect the emergence of one $C$ atom to the surface each $\mu$ s 
per $\AA^{2}$, and lasting at the surface for a substantial time, almost $1 \mathrm{~ms}$ in the case of $\mathrm{Ag}$. Obviously, a frequent and long $\mathrm{C}$ presence can clearly condition the surface activity and the on-going processes, which underlines the $\mathrm{C}$ subsurface chemistry as a factor to be considered when working with $\mathrm{Ni}, \mathrm{Cu}, \mathrm{Pd}$, and $\mathrm{Ag}$ surfaces, especially critical in the case of $\mathrm{Ag}$, as an apparently clean surface at $300 \mathrm{~K}$ could contain $\mathrm{C}$ trenched subsurface that may emerge when the surface is heated at the working conditions.

For the systems where $\mathrm{C}$ is found to prefer surface sites ( $\mathrm{Rh}, \mathrm{Ir}, \mathrm{Pt}$, and $\mathrm{Au})$ the presence of subsurface $\mathrm{C}$ might not critically condition the on-going catalysis, as the main influence will come from surface $\mathrm{C}$ adatoms. In these cases, the only effect of subsurface $\mathrm{C}$ presence would be to facilitate the mobility of carbon around the lattice and, eventually, emerge on C-free or C-containing areas of the surface; i.e. it could open another channel to move $\mathrm{C}$ contamination to all possible sites in the lattice. Moreover, the emergence of subsurface $\mathrm{C}$ atom may imply another front of attack in the catalytic reaction, which can be important e.g. on $\mathrm{Pt}$, given then enhanced $\mathrm{C}$ solubility, see Figure 4. For Ir, however, the presence of subsurface C, only detectable at high temperatures, is merely testimonial. Not only the sinking processes are rarely occurring, but also the diffusion at surface, with time scales above the $\mu$ s even at $700 \mathrm{~K}$. Rh partially populates subsurface at the equilibrium at $700 \mathrm{~K}$, but the $l t_{\text {surf }}$ is above the seconds timeframe even at $700 \mathrm{~K}$, which implies that, even if subsurface is partially populated, such carbon atoms would be trapped inside, neither moving nor emerging to other surface areas. However, they can locally perturb the electronic density of the Rh metal atoms immediately above, and, eventually, their chemical activity and reactivity.

For $\mathrm{Au}, \mathrm{kMC}$ simulations predict a substantial reduction of both $\bar{t}_{e m}$ and $l t_{r}$, similar to Pt. This translates into a much more dynamic equilibrium as compared to the other cases, with a continuous exchange of $\mathrm{C}$ atoms between surface and subsurface, especially at $700 \mathrm{~K}$, where this exchange is below the ps time scale. As shown in Figure 4 , however, the subsurface population only reaches considerable values for Pt, slightly above $10 \%$ of the total carbon at $700 \mathrm{~K}$, while only $2-3 \%$ for Au. Surface C moieties, then, are anyway expected, yet the extra disorder effect caused by the opening of the subsurface diffusion channel will certainly be present in Au and mainly in Pt surfaces when exposed to low coverages of surface C.

\section{Conclusions}


By means of $\mathrm{kMC}$ simulations with rates derived from periodic DFT calculations and TST, the present work assesses the dynamic mobility of $\mathrm{C}$ atoms on all fcc TM (111) surfaces under working conditions of low $C$ coverage; $\theta_{\mathrm{C}}=1-9$ at $\%$ and working temperatures ranging 300-700 K. Overall, the macroscopic kinetic properties obtained from the $\mathrm{kMC}$ simulations are in agreement with the predictions made from the static DFT energy landscape. For instance, kMC simulations show that at $300 \mathrm{~K} \mathrm{C}$ atoms spend most of the time around the thermodynamically most stable adsorption sites, with little effect of the $\theta_{\mathrm{C}}$. These sites are subsurface oss for $\mathrm{Ni}, \mathrm{Cu}, \mathrm{Pd}$, and $\mathrm{Ag}$, and surface $f c c$ for $\mathrm{Rh}, \mathrm{Ir}, \mathrm{Pt}$, and $\mathrm{Au}$.

In some cases, however, other adsorption sites start to become populated at higher temperatures. For instance, subsurface sites for Pt and $\mathrm{Rh}$, and surface sites for Ag. This is an unexpected conclusion, which possibly cannot be anticipated by inspection of the DFT energy profiles alone, and that may have relevant impact in the catalytic activity of these transition metal surfaces at high temperature. Present kMC simulations also show that, in general, surface and subsurface diffusions dominate the kinetics, with sinking/emerging processes being much less frequent, and, surprisingly, occurring involving the channel connecting surface $h c p$ and subsurface tss sites. In the case of Ag, and to a lesser extent $\mathrm{Pd}$, emerging processes are triggered by annealing, and the thus raised $\mathrm{C}$ atoms can vary or bias on-going catalytic processes occurring on these surfaces. For Au and Pt, C mobility has been seen to be considerably higher than for the rest of the studied surfaces. Even if the $\mathrm{C}$ is mainly found at surface, it can diffuse through the subsurface region to other areas, either $\mathrm{C}$-free or C-containing, and even imply a new reaction mechanism with a $\mathrm{C}$ attack from subsurface. Even though the study is focused on (111) $f c c$ surfaces, it is likely that such effects can be present in other surface terminations, even in TMs with different crystallographic structure. The present results highlight the need of reconsidering the role of both surface and subsurface carbon moieties in the simulation models, a factor which has been, hitherto, mostly neglected.

\section{Acknowledgements.}

The present work was financially supported by the Spanish Ministerio de Ciencia $y$ Universidades (MICIUN) RTI2018-095460-B-I00 and María de Maeztu MDM-20170767 grants and, in part, by Generalitat de Catalunya (grants 2017SGR13 and 
XRQTC). O.P. thanks the Spanish MICIUN for a PhD grant (PRE2018-083811). F.I. acknowledges additional support from the 2015 ICREA Academia Award for Excellence in University Research. 
Table 1. Total number of carbon atoms, $N_{\mathrm{C}}$, and lattice size $p(N \times N)$, and total number of sites, $n_{\text {sites }}$, as a function of $\theta_{\mathrm{C}}$.

\begin{tabular}{cccccc}
\hline & \multicolumn{5}{c}{$\boldsymbol{\theta}_{\mathbf{C}} / \mathbf{a t} \%$} \\
& $\mathbf{1}$ & $\mathbf{3}$ & $\mathbf{5}$ & $\mathbf{7}$ & $\mathbf{9}$ \\
\hline \hline $\boldsymbol{n}_{\boldsymbol{C}}$ & 98 & 150 & 160 & 126 & 162 \\
$\boldsymbol{p}(\boldsymbol{N} \times \boldsymbol{N})$ & $p(70 \times 70)$ & $p(50 \times 50)$ & $p(40 \times 40)$ & $p(30 \times 30)$ & $p(30 \times 30)$ \\
$\boldsymbol{n}_{\text {sites }}$ & 19600 & 10000 & 6400 & 3600 & 3600 \\
\hline
\end{tabular}


Figure 1. Schematic representation of the employed lattice unit cell indicating the considered active sites, and their connectivity; a) representation of the adsorption sites included in the unit cell, and the eight different diffusion processes accounted for in the $\mathrm{kMC}$ simulations, b) top view of a $p(2 \times 2)$ supercell. Black spheres denote metal atoms, and the unit cell units are represented with black lines. Colored smaller (larger) spheres denote surface (subsurface) sites following the notation in a), and colored lines show the connectivity between sites within the same layer, and c) side view of b), where colored lines show the sinking/emerging channels of the lattice.

a)

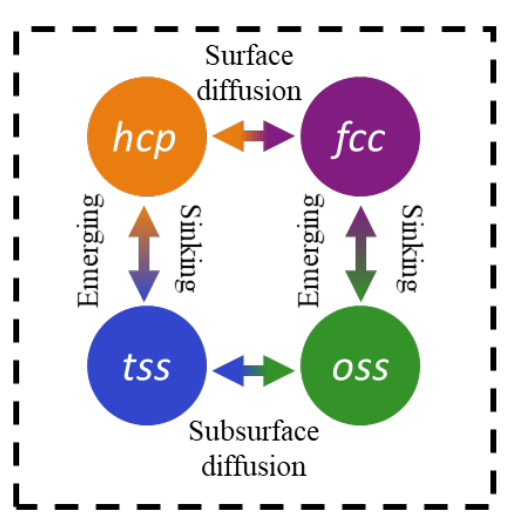

b)

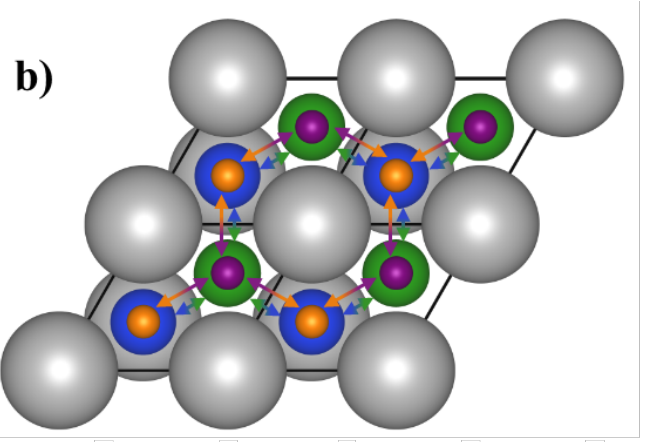

c)

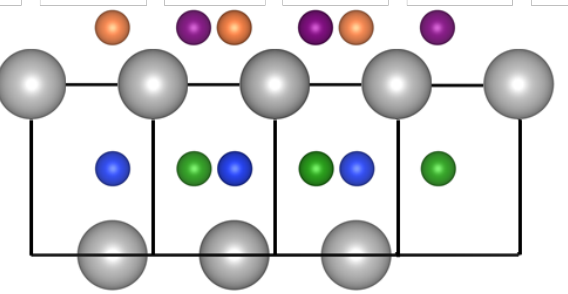


Figure 2. Energy profiles for $h c p \leftrightarrow f c c$ surface and $t s s \leftrightarrow o s s$ subsurface diffusions of an isolated $\mathrm{C}$ atom for the eight studied $f c c$ transition metal (111) surfaces. The left topmost panel contains a description of the processes and indicates the meaning of the numerical values in the rest of panels.

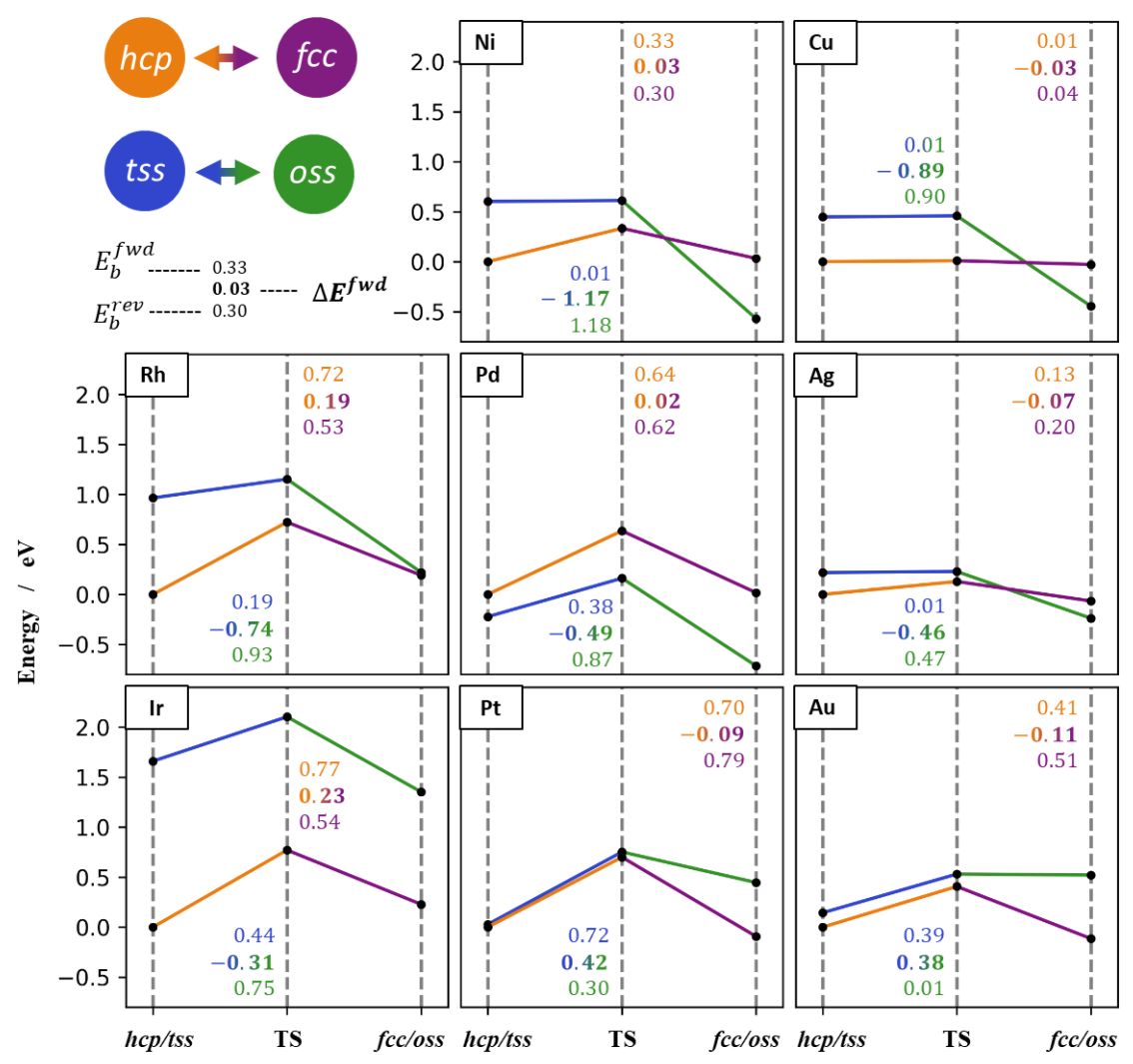


Figure 3. Energy profiles for $h c p \leftrightarrow t s s$ and $f c c \leftrightarrow o s s$ sinking/emerging processes of an isolated $\mathrm{C}$ atom for the eight studied $f c c$ transition metal (111) surfaces. Diffusion energy barriers were initially obtained from literature [14] but here include the ZPE term. The topmost left panel is as in Figure 2.

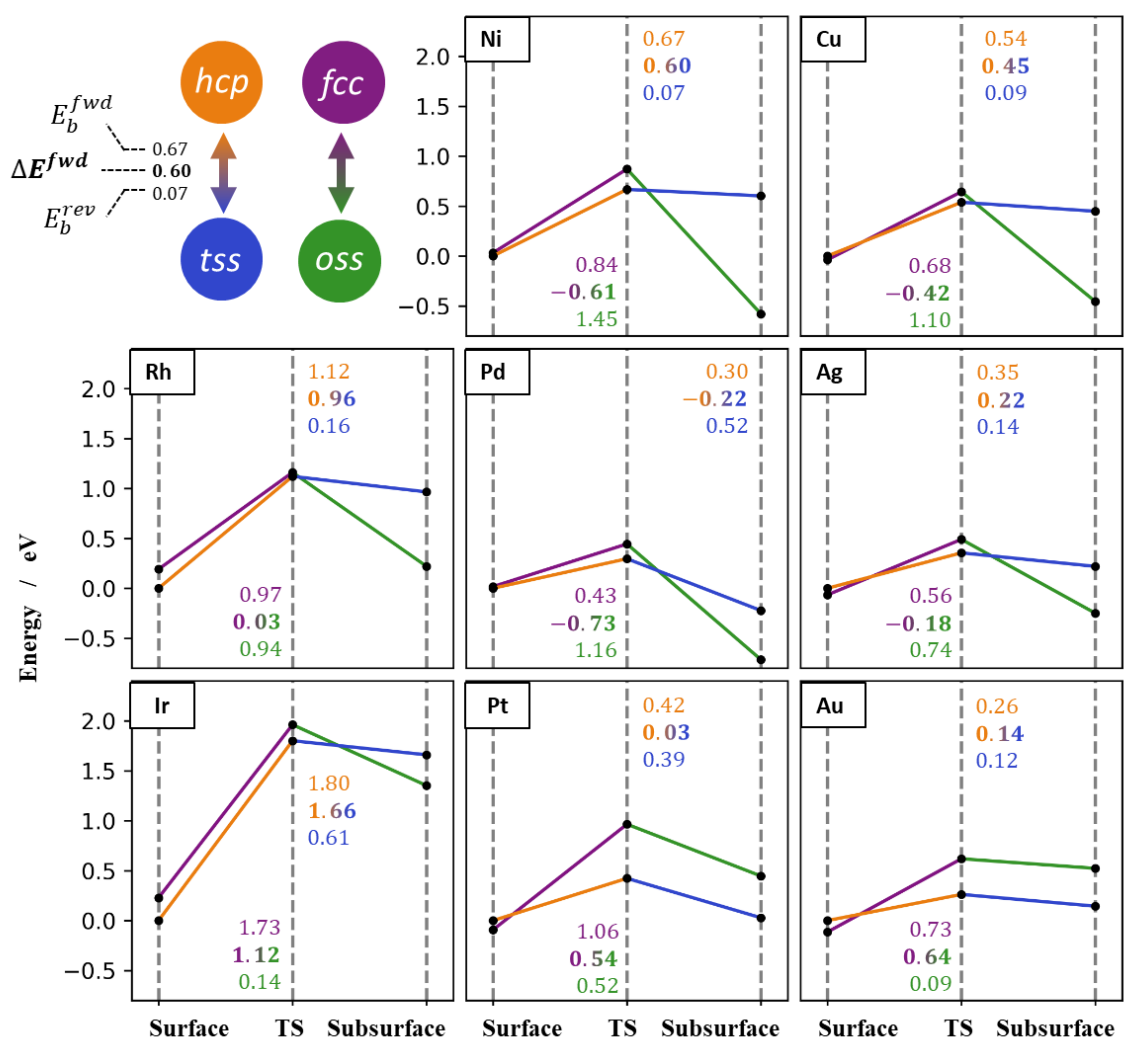


Figure 4. Percentage subsurface $\mathrm{C}$ for the studied $f c c$ TM (111) surfaces as a function of the total $\theta_{\mathrm{C}}$ and in the temperature ranges of $300-700 \mathrm{~K}$.
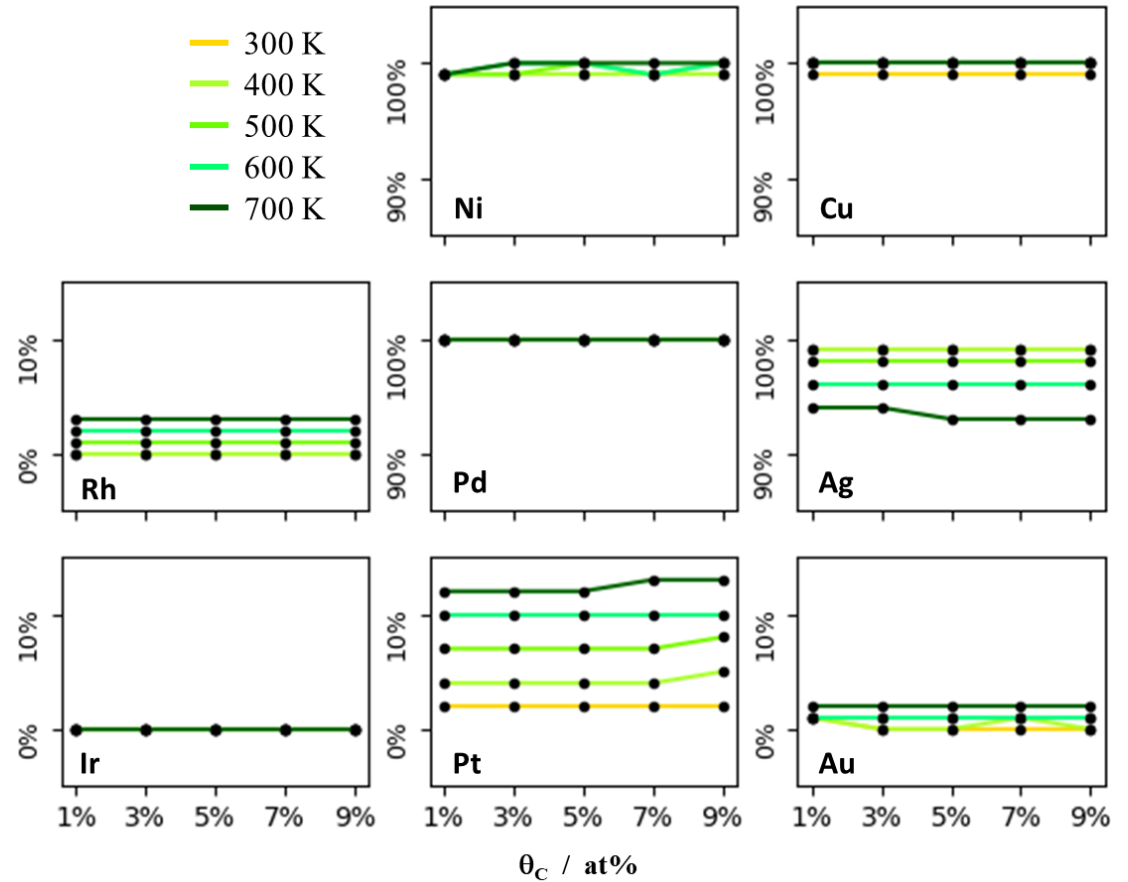
Figure 5. Mean lifetime of a $\mathrm{C}$ atom on the or subsurface regions as a function of temperature, $T$. All values have been computed for $\theta_{\mathrm{C}}=1$ at $\%$. Note that for Ir no subsurface sites were populated below $600 \mathrm{~K}$ in the performed simulations.

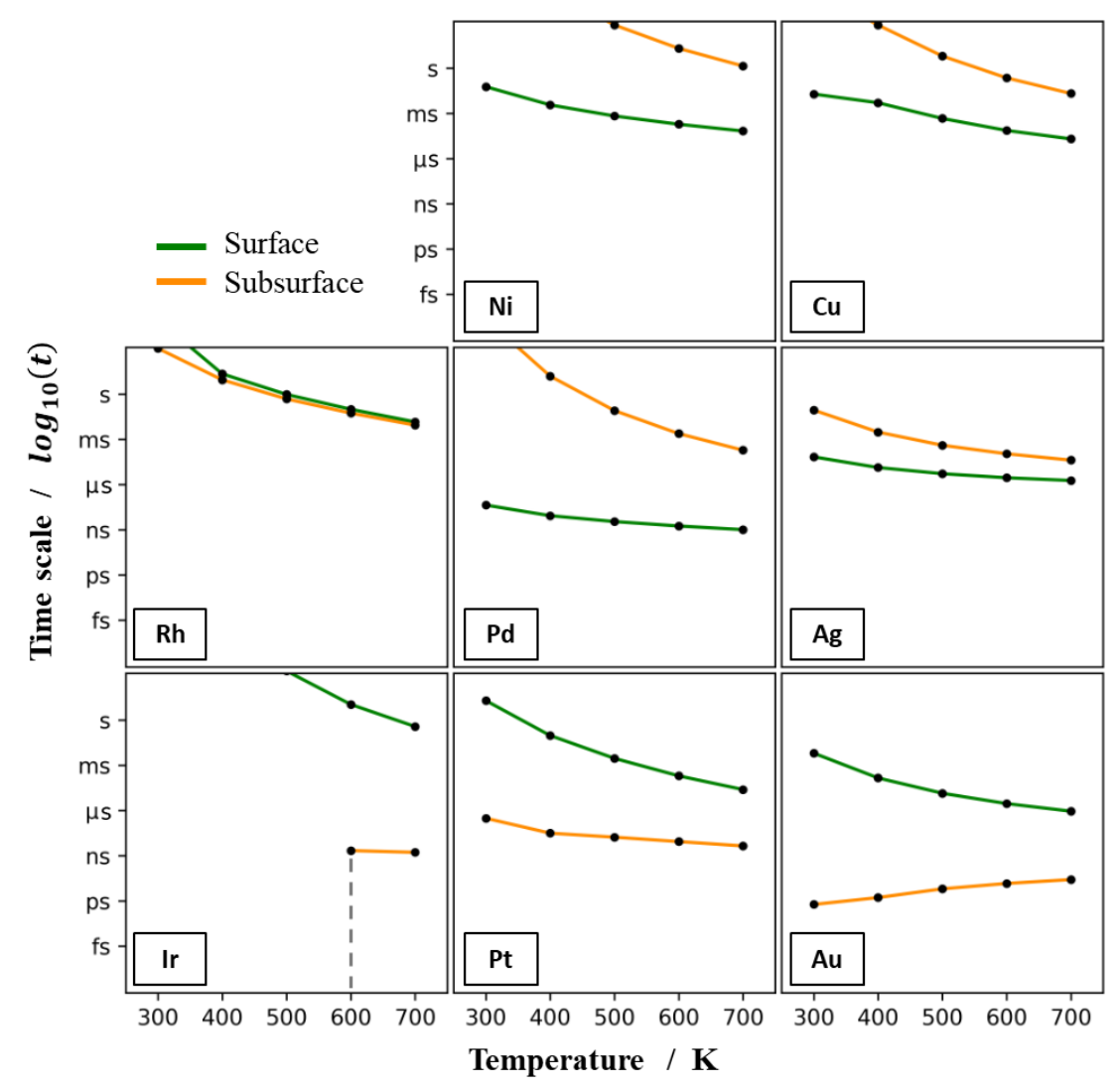


Figure 6. Mean lifetime of $\mathrm{C}$ in the different regions; $l t_{\text {surf }}$ and $l t_{\text {sub }}$, and in the four different adsorption/absorption sites, $l t_{f c c}, l t_{h c p}, l t_{t s s}$, and $l t_{o s s}$, as well as the average waiting time between two consecutive emerging processes through either $f c c, \bar{t}_{e m}^{f c c}$, or $h c p, \bar{t}_{e m}^{h c p}$. All values have been computed at $300 \mathrm{~K}$ (dark grey) and $700 \mathrm{~K}$ (light grey), and $\theta_{\mathrm{C}}=1 \mathrm{at} \%$. For $l t_{\text {surf }}$ and $l t_{\text {sub }}$, a decomposition in terms of $h c p / t s s$ and fcc/oss sites is provided in light blue and orange insight each grey bar. See the SM for further details on the interpretation of the bars.

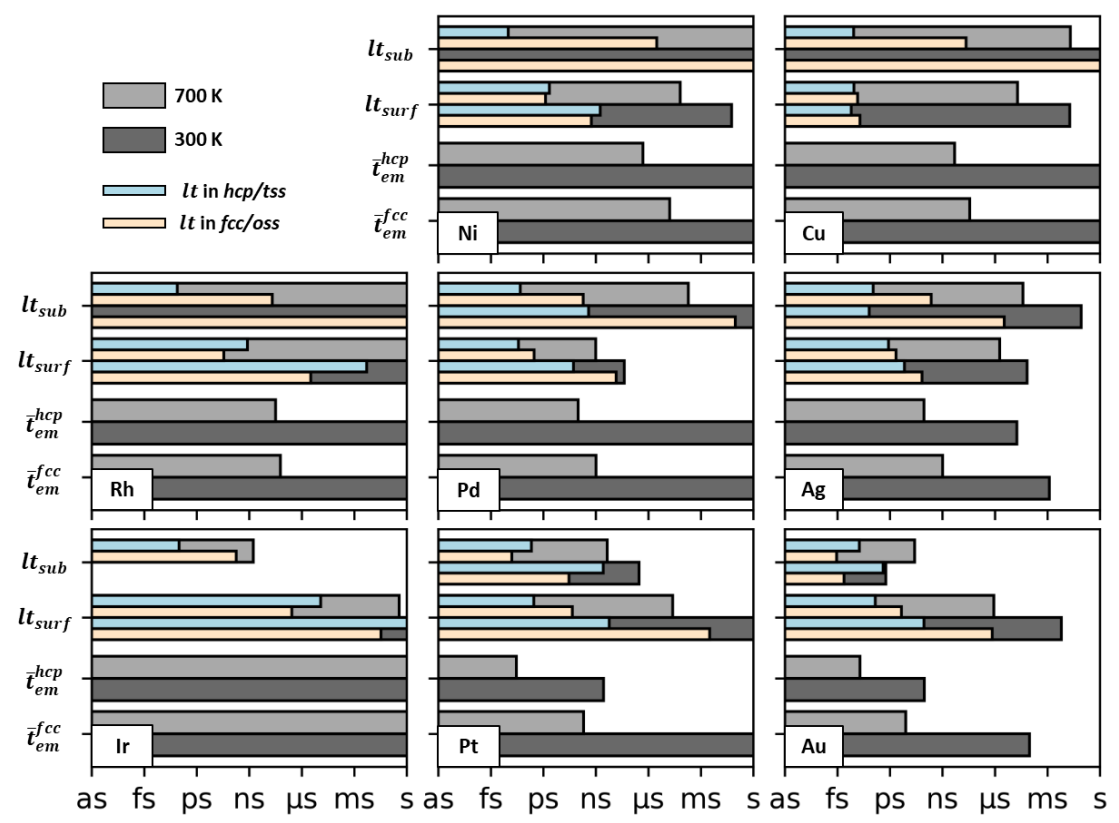




\section{References}

[1] J.W. Erisman, M.A. Sutton, J. Galloway, Z. Klimont, W. Winiwarter, How a century of ammonia synthesis changed the world, Nat. Geosci. 1 (2008) 636-639.

[2] L. Vega, B. Martínez, F. Viñes, F. Illas, Robustness of surface activity electronic structurebased descriptors of transition metals, Phys. Chem. Chem. Phys. 20 (2018) 20548-20554.

[3] A.J. Medford, A. Vojvodic, J.S. Hummelshøj, J. Voss, F. Abild-pedersen, F. Studt, T. Bligaard, A. Nilsson, J.K. Nørskov, From the Sabatier principle to a predictive theory of transition-metal heterogeneous catalysis, J. Catal. 328 (2015) 36-42.

[4] H.F. Abbas, M.A.W. Wan Daud, Hydrogen production by methane decomposition: A review, Int. J. Hydrog. Energy. 35 (2010) 1160-1190.

[5] J. Van Houten, A century of chemical dynamics traced through the nobel prizes. 1909: Wilhelm Ostwald, J. Chem. Educ. 79 (2002) 146-148.

[6] K.J. Smith, R.B. Anderson, The higher alcohol synthesis over promoted $\mathrm{Cu} / \mathrm{ZnO}$ catalysts. Can. J. Chem. Eng. 61 (1983) 40-45.

[7] P.D.F. Vernon, M.L.H. Green, A.K. Cheetham, A.T. Ashcroft, Partial oxidation of methane to synthesis gas. Catal. Lett. 6 (1990) 181-186.

[8] C.H. Bartholomew, Carbon deposition in steam reforming and methanation, Catal. Rev.-Sci. Eng. 24 (1982) 67-112.

[9] H. Ren, M.P. Humbert, C.A. Menning, J.G. Chen, Y. Shu, U.G. Singh, W.C. Cheng, Inhibition of coking and $\mathrm{CO}$ poisoning of $\mathrm{Pt}$ catalysts by the formation of $\mathrm{Au} / \mathrm{Pt}$ bimetallic surfaces, Appl. Catal., A. 375 (2010) 303-309.

[10] P. Albers, J. Pietshc, S.F. Parker, Poisoning and deactivation of palladium catalysts, J. Mol. Catal. A Chem. 173 (2001) 275-286.

[11] J.C. Rodríguez, E. Romeo, J.L.G. Fierro, J. Santamaría, A. Monzón, Deactivation by coking and poisoning of spinel-type Ni catalysts, Catal. Today. 37 (1997) 255-265.

[12] R.T.K. Baker, M.A. Barber, P.S. Harris, F.S. Feates, R.J. Waite, Nucleation and growth of carbon deposits from the nickel catalyzed decomposition of acetylene, J. Catal. 26 (1972) 5162.

[13] C.J. Weststrate, A.C. Kazalkaya, E.T.R. Rossen, M.W.G.M. Verhoeven, I.M. Ciobîcă, A.M. Saib, J.W. Niemantsverdriet, Atomic and polymeric carbon on Co(0001): Surface reconstruction, graphene formation, and catalyst poisoning, J. Phys. Chem. C. 116 (2012) $11575-11583$.

[14] O. Piqué, I.Z. Koleva, F. Viñes, H.A. Aleksandrov, G.N. Vayssilov, F. Illas, Subsurface carbon: A general feature of noble metals, Angew. Chem. Int. Ed. 58 (2019) 1744-1748. [15] P. Janthon, F. Viñes, J. Sirijaraensre, J. Limtrakul, F. Illas, Carbon dissolution and segregation in platinum, Catal. Sci. Technol. 7 (2017) 807-816. 
[16] D. Teschner, J. Borsodi, A. Wootsch, Z. Révay, M. Hävecker, A. Knop-Gericke, S.D. Jackson, R. Schlögl, The roles of subsurface carbon and hydrogen in palladium-catalyzed alkyne hydrogenation, Science (80-. ). 320 (2008) 86-89.

[17] A.J. Du, S.C. Smith, X.D. Yao, G.Q. Lu, Catalytic effects of subsurface carbon in the chemisorption of hydrogen on a $\operatorname{Mg}(0001)$ surface: An ab-initio study, J. Phys. Chem. B. 110 (2006) 1814-1819.

[18] G. Kresse, J. Furthmüller, Efficient iterative schemes for ab initio total-energy calculations using a plane-wave basis set, Phys. Rev. B. 54 (1996) 169-186.

[19] J.P. Perdew, K. Burke, M. Ernzerhof, Generalized gradient approximation made simple, Phys. Rev. Lett. 77 (1996) 3865-3868.

[20] J. Paier, M. Marsman, G. Kresse, Why does the B3LYP hybrid functional fail for metals?, J. Chem. Phys. 127 (2007) 1-10.

[21] L. Vega, J. Ruvireta, F. Viñes, F. Illas, Jacob's ladder as sketched by Escher: Assessing the performance of broadly used density functionals on transition metal surface properties, J. Chem. Theory Comput. 14 (2018) 395-403.

[22] P. Janthon, S. M. Kozlov, F. Viñes, J. Limtrakul, and F. Illas, J. Chem. Theory Comput. 9 (2013) 1631-1640

[23] P. Janthon, S. Luo, S. M. Kozlov, F. Viñes, J. Limtrakul, D. G. Truhlar, F. Illas, J. Chem. Theory Comput., 10 (2014) 3832-3839

[24] J. Ruvireta, L. Vega, F. Viñes, Cohesion and coordination effects on transition metal surface energies, Surf. Sci. 664 (2017) 45-49.

[25] G. Henkelman, U. Blas P., H. Jónsson, A climbing image nudged elastic band method for finding saddle points and minimum energy paths and minimum energy paths, J. Chem. Phys. 113 (2000) 9901-9904.

[26] H.J. Monkhorst, J.D. Pack, Special points for Brillouin-zone intergrations, Phys. Rev. B. 13 (1976) 5188-5192.

[27] A.P.J. Jansen, An introduction to kinetic Monte Carlo simulations of surface reactions, Lecture Notes in Physics, vol. 856, Springer-Verlag, Heidelberg, Germany, 2012.

[28] H. Prats, S. Posada-Pérez, J.A. Rodriguez, R. Sayós, F. Illas, Kinetic monte carlo simulations unveil synergic effects at work on bifunctional catalysts, ACS Catal. 9 (2019) 9117-9126.

[29] L. Yang, A. Karim, J.T. Muckerman, Density functional kinetic monte carlo simulation of water-gas shift reaction on $\mathrm{Cu} / \mathrm{ZnO}$, J. Phys. Chem. C. 117 (2013) 3414-3425.

[30] S. Piccinin, M. Stamatakis, CO oxidation on Pd(111): A first-principles-based kinetic monte carlo study, ACS Catal. 4 (2014) 2143-2152. 
[31] H. Prats, L. Álvarez, F. Illas, R. Sayós, Kinetic monte carlo simulations of the water gas shift reaction on $\mathrm{Cu}(111)$ from density functional theory based calculations, J. Catal. 333 (2016) $217-226$.

[32] A.U. Nilekar, J. Greeley, M. Mavrikakis, A simple rule of thumb for diffusion on transition-metal surfaces, Angew. Chem. Int. Ed. 45 (2006) 7046-7049.

[33] F. Viñes, C. Loschen, F. Illas, K.M. Neyman, Edge sites as a gate for subsurface carbon in palladium nanoparticles, J. Catal. 266 (2009) 59-63.

[34] D. Teschner, J. Borsodi, A. Wootsch, Z. Révay, M. Hävecker, A. Knop-Gericke, S.D. Jackson, R. Schlögl, The roles of subsurface carbon and hydrogen in Palladium-catalyzed alkyne hydrogenation, Science, 320 (2008) 86-89.

[35] A. Rinaldi, J.-P. Tessonnier, M.E. Schuster, R. Blume, F. Girgsdies, Q. Zhang, T. Jacob, S. B. Abd Hamid, D.S. Su, R. Schlögl, Dissolved carbon controls the initial stages of nanocarbon growth, Angew. Chem. Int. Ed. 50 (2011) 3313-3317.

[36] H. Zi-Pu, D.F. Ogeltree, M.A. van Hove, G.A. Somorjai, Leed theory for incommensurate overlayers: Application to graphite on Pt(111), Surf. Sci. 180 (1987) 433-459.

[37] F. Viñes, K.M. Neyman, A. Görling, Carbon on Platinum substrates: From carbidic to graphitic phases on the (111) surface and on nanoparticles, J. Phys. Chem. A, 2009, 113, 11963-11973. 